\title{
Role of endometriosis fertility index system in predicting non-IVF conception in patients with surgically documented endometriosis
}

\author{
Fessy Louis T. ${ }^{1}$, Deepa Naveen ${ }^{2}$, Ramesh P. ${ }^{*}$, Parasuram Gopinath $^{2}$, Gopinathan K. K. ${ }^{2}$
}

\begin{abstract}
${ }^{1}$ Department of Reproductive Medicine and Surgery, Amrita Institute of Medical Sciences, Kochi, India
${ }^{2}$ Department of Reproductive Medicine, CIMAR, Edappal Hospitals Pvt Ltd, Edappal, Kerala, India
\end{abstract}

\author{
Received: 28 March 2019 \\ Accepted: 06 May 2019

\section{*Correspondence:} \\ Dr. Ramesh P., \\ E-mail: docrameshp@gmail.com
}

Copyright: (C) the author(s), publisher and licensee Medip Academy. This is an open-access article distributed under the terms of the Creative Commons Attribution Non-Commercial License, which permits unrestricted non-commercial use, distribution, and reproduction in any medium, provided the original work is properly cited.

\begin{abstract}
Background: Endometriosis still remains an enigmatic disease. There are important reasons to stage endometriosis and to prognosticate the chances of pregnancy after a surgical management. The currently used revised AFS system has poor correlation with pregnancy rate. A scoring system-Endometriosis fertility index (EFI) to prognosticate the outcome was proposed few years back. The objective was to assess the usefulness of the EFI system in predicting pregnancy in patients with surgically documented endometriosis who attempt Non-IVF conception.

Methods: Retrospective data was collected from 77 subjects with endometriosis who underwent laparoscopy and had documented least function (LF) score and EFI score. All were followed up until 12 months for the occurrence of a non IVF pregnancy.

Results: Our study showed that the pregnancy rate was clearly higher in those with high EFI scores than those with low scores. A score of less than 4 was associated with significantly lower pregnancy rates than those with score above $5(\mathrm{n}=26$, pregnancy rate- $11.54 \%)$ vs. $(\mathrm{n}=51$, pregnancy rate $50.1 \%) ; \mathrm{p}=0.001)$ ). Similarly, the pregnancy rate was significantly lower in those with LF score 1-3 (21.2\%) as opposed to those with higher LF scores $(p=0.029)$. Also, sensitivity analysis showed that higher EFI score was significantly associated with higher LF score $(\mathrm{P}<0.001)$.

Conclusions: EFI is a useful clinical tool that predicts pregnancy with reasonable accuracy after endometriosis surgery. Its use clearly provides reassurance to those patients with good prognosis.
\end{abstract}

Keywords: Endometriosis fertility index, Laparoscopy, Least function score, Prognosis, Staging

\section{INTRODUCTION}

In 1979 , the American fertility society (AFS) (now the American society for Reproductive Medicine) first proposed a classification system for endometriosis. ${ }^{1}$ This was extensively evaluated modified in $1985 .^{2-5}$ Despite these revisions the currently used revised AFS system has serious limitations. ${ }^{6-13}$ Chief among them is the relatively poor correlation with pregnancy rates. ${ }^{14}$ In 2009 a new staging system Endometriosis Fertility Index was devised with (EFI) score from 0-10, 0 - representing poorest prognosis and 10- representing the best prognosis. ${ }^{15}$ Key element of the new staging system was a numerical measure of functional anatomy based on careful assessment of tubes (extent of serosal injury, mobility and patency) fimbria (extent of injury and architecture) ovaries (size and extent of surface injury). Other aspects like age, duration of infertility and history of prior pregnancy were evaluated. The pregnancy rate reported after the utilization of the new scoring system was showing conflicting results. The usefulness of such a system in other ethnic populations also is less studied. 
Our objective was to assess the usefulness of the EFI system in predicting pregnancy in patients with surgically documented endometriosis who attempt non-ART conception.

\section{METHODS}

This study was carried out from January 2018 to December 2018. This was a retrospective study including 77 patients between 21-40 years who underwent Laparoscopic surgery for endometriosis at our centre from 2013 to 2017. Authors included those couples with normal male factor as indicated by semen analysis according to WHO 2010 criteria. All female partners had undergone laparoscopy for endometriosis and had documented LF score and AFI score. The records were searched for occurrence of a non ART conception. Those who underwent second surgery for endometriosis were excluded. We also excluded those who were recruited for In vitro fertilization (IVF/ ICSI).

As part of institutional protocol, the initial survey included a routine gynecological examination, the relevant results of previous diagnostic tests were noted. Baseline trans vaginal ultrasound was done for all subjects. The patients with endometriosis who might be benefitted from surgical management were subjected to laparoscopy and the findings were noted. These were mapped based on EFI scoring system (proposed by Adamson et al) as described below. ${ }^{15}$

Table 1: Description of least function score.

\begin{tabular}{|c|c|c|}
\hline Structure & Dysfunction & Description \\
\hline \multirow[t]{4}{*}{ Tube } & Mild & Slight injury to serosa of the fallopian tube \\
\hline & Moderate & $\begin{array}{l}\text { Moderate injury to serosa or muscularis of the fallopian tube; moderate limitation in } \\
\text { mobility }\end{array}$ \\
\hline & Severe & $\begin{array}{l}\text { Fallopian tube fibrosis or mild/moderate salpingitis isthmica nodosa; severe limitation in } \\
\text { mobility }\end{array}$ \\
\hline & Nonfunctional & Complete tubal obstruction, extensive fibrosis or salpingitis isthmica nodosa \\
\hline \multirow[t]{4}{*}{ Fimbria } & Mild & Slight injury to fimbria with minimal scarring \\
\hline & Moderate & $\begin{array}{l}\text { Moderate injury to fimbria, with moderate scarring, moderate loss of fimbrial architecture } \\
\text { and minimal intrafimbrial fibrosis }\end{array}$ \\
\hline & Severe & $\begin{array}{l}\text { Severe injury to fimbria, with severe scarring, severe loss of fimbrial architecture and } \\
\text { moderate intrafimbrial fibrosis }\end{array}$ \\
\hline & Nonfunctional & $\begin{array}{l}\text { Severe injury to fimbria, with extensive scarring, complete loss of fimbrial architecture, } \\
\text { complete tubal occlusion or hydrosalpinx }\end{array}$ \\
\hline \multirow[t]{4}{*}{ Ovary } & Mild & Normal or normal ovarian size; minimal or mild injury to ovarian serosa \\
\hline & Moderate & Ovarian size reduced by one-third or more; moderate injury to ovarian surface \\
\hline & Severe & Ovarian size reduced by two-thirds or more; severe injury to ovarian surface \\
\hline & Nonfunctional & Ovary absent or completely encased in adhesions \\
\hline
\end{tabular}

Least Function Score, the functional score was determined for each Tube, fimbria, and ovary bilaterally, 0=Nonfunctional/Absent; 1=Severe; $2=$ Moderate; $3=$ Mild dysfunction; 4=Normal.

Scores for the tube, fimbria, and ovary were combined by summing or taking the minimum, separately by side and for both sides combined. One such composite score was the least function scores- the sum of lowest function score on each side from among the fallopian tube, fimbria, and ovary. Score of 4 could be obtained on one side only if the tube, fimbria, ovary each were entirely normal, score of 0 on one side could be obtained if the tube was absent, obstructed proximally, completely fibrotic, completely encased in dense adhesion; if the fimbria was involved in a hydrosalpinx, was completely fibrotic, or was separated from the ovary by dense adhesion that had not been removed or if the ovary was surgically or otherwise absent, or completely encased in dense adhesion such that an egg could not enter the fallopian tube .
The total least function score was obtained by adding the lowest score from the right side to the lowest score from the left side to give a combined total of potential for reproductive function in the pelvis. Completely normal pelvis would have a score of $4+4=8$ and have excellent reproductive potential. Completely nonfunctional pelvis with no chance of reproductive potential have a score of $0+0=0$.

If the ovary is absent on one side, all the ovulation will occur from the ovary on the other side. Therefore in this situation the least function score was obtained by determining the function score on the side with the ovary and then doubling it. 
The result of abdominal surgery were recorded in substantial detail as

- Revised American fertility scoring system

- Percentage of filmy and dense adhesion on the ovaries and tubes bilaterally.

These intra operative scoring system was considered supplement's to the historical factors that predicted pregnancy rate (age, duration of infertility and pregnancy history)

The detail of the scoring system is depicted in Figure 1. The final score was noted. All were followed up for at least 12 months. Any non ART conceptions (Spontaneous/ Ovulation induction \pm IUI) were noted down. The percentage of occurrence of pregnancies were tabulated against the EFI scores and were analysed statistically to see if there are any correlations between EFI score and pregnancy.

\section{Statistical analysis}

Statistical analysis was done using IBM SPSS software (version 17). The significance was assessed at 5\% level and $\mathrm{p}$ value of $<0.05$ was considered statistically significant.

\section{RESULTS}

The basal and demographic characteristics of the study population are depicted in Table 2 . The mean age was $32.8 \pm 4.7$ years. Majority $(66 \%)$ had primary infertility.

Table 2: Basal and demographic characteristics.

\begin{tabular}{|ll|}
\hline Parameter & Value- mean (SD) / number (percentage) \\
\hline Age (years) & $32.8(4.7)$ \\
\hline BMI & $24.8(4.07)$ \\
\hline Primary infertility & $51(66.2 \%)$ \\
\hline Duration of infertility (years) & $4.08(1.96)$ \\
\hline AMH (ng/ml) & $3.46(1.88)$ \\
\hline Day 2 FSH (IU/L) & $6.27(2.55)$ \\
\hline Conception rate at 1year follow up & $29(37.66 \%)$ \\
\hline
\end{tabular}

BMI; Body mass index, AMH; Ante mullerian hormone, FSH; Follicle stimulating hormone

Table 3: Distribution of pregnancy in relation with EFI score.

\begin{tabular}{|llll|}
\hline EFI score & Number of patients & Number of positive pregnancy & Pregnancy rate (\%) \\
\hline $0-3$ & 17 & 2 & 11.8 \\
\hline 4 & 9 & 1 & 11.1 \\
\hline 5 & 8 & 3 & 37.5 \\
\hline 6 & 8 & 5 & 62.5 \\
\hline 7 & 13 & 6 & 46.2 \\
\hline 8 & 6 & 4 & 66.7 \\
\hline $9-10$ & 16 & 8 & 50.0 \\
\hline Total & 77 & 29 & 37.7 \\
\hline
\end{tabular}

EFI; Endometriosis fertility index score

Table 4: Distribution of pregnancy rate in relation with LF score.

\begin{tabular}{|c|c|c|c|c|}
\hline LF score & $\begin{array}{l}\text { Number of patients } \\
(n=77)\end{array}$ & $\begin{array}{l}\text { Number of positive } \\
\text { pregnancy }\end{array}$ & $\begin{array}{l}\text { Pregnancy } \\
\text { rate }(\%)\end{array}$ & P value \\
\hline Group I (7-8) & 28 & 13 & 46.4 & \multirow{3}{*}{$0.029 *$} \\
\hline Group II (4-6) & 16 & 9 & 56.3 & \\
\hline Group III $(1-3)^{¥}$ & 33 & 7 & 21.2 & \\
\hline
\end{tabular}

LF score= Least function score; Maximum pregnancy was seen in those groups with low LF scores; ¥ Group I vs Group II- $\mathrm{p}=0.56$, Group I vs Group III - $\mathrm{p}=0.038$, Group II vs Group III- $\mathrm{p}=0.01$

Our study showed that the pregnancy rate was clearly high in those with high EFI scores than those with low scores (Table 3). A score of less than 4 was associated with significantly lower pregnancy rates than those with 
score above $5[(\mathrm{n}=26$, pregnancy rate- $11.54 \%)$ vs. $(\mathrm{n}=51$, pregnancy rate $50.1 \%) ; \chi 2=10.83, \mathrm{DF}=1, \mathrm{p}=0.001)]$.

Similarly, the pregnancy rate was significantly lower (Table 4) in those with LF score 1-3 $(21.2 \%)$ as opposed to those with higher LF scores $(\mathrm{p}=0.029)$. The subgroup analysis revealed that there was no statistically significant difference between LF scores of 7-8 and 4-6 $(\mathrm{p}=0.56)$. However, the low LF score group (1-3) had significantly different pregnancy rate as opposed to medium score (46 ) and high score (7-8). The correlation of EFI score and LF score was analyzed. The mean LF score was $2.12 \pm 0.48$ in those with EFI score $0-3$ as compared to $7.63 \pm 0.50$ in those with EFI score 9-10. A sensitivity analysis was performed to assess the effect of the potential differences in the assignment of the LF score on the EFI. The results showed that higher EFI score was significantly associated with higher LF score $(\mathrm{P}<0.001)$.

Table 5: Correlation of EFI score and pregnancy rate - comparison with previous studies.

\begin{tabular}{|llllll|} 
EFI score & $\begin{array}{l}\text { Number of } \\
\text { patients }\end{array}$ & $\begin{array}{l}\text { Number of } \\
\text { positive } \\
\text { pregnancy }\end{array}$ & $\begin{array}{l}\text { Cum Pregnancy rate } \\
\text { (present study) } \\
\text { (18 months) }\end{array}$ & $\begin{array}{l}\text { (David Adamson et al) } \\
\text { Cum pregnancy } \\
\text { rate (12 months) }\end{array}$ & $\begin{array}{l}\text { Cum pregnancy } \\
\text { rate (24 months) }\end{array}$ \\
\hline $0-3$ & 17 & 2 & 11.76 & 9.9 & 9.9 \\
\hline 4 & 26 & 3 & 11.54 & 15.2 & 23.2 \\
\hline 5 & 34 & 6 & 17.65 & 22.8 & 38.8 \\
\hline 6 & 42 & 11 & 26.19 & 29.5 & 48.0 \\
\hline 7 & 55 & 17 & 30.91 & 37.4 & 57.8 \\
\hline 8 & 61 & 21 & 34.43 & 41.0 & 57.9 \\
\hline $9-10$ & 77 & 29 & 37.66 & 56.4 & 71.9 \\
\hline
\end{tabular}

EFI score- Endometriosis fertility index score

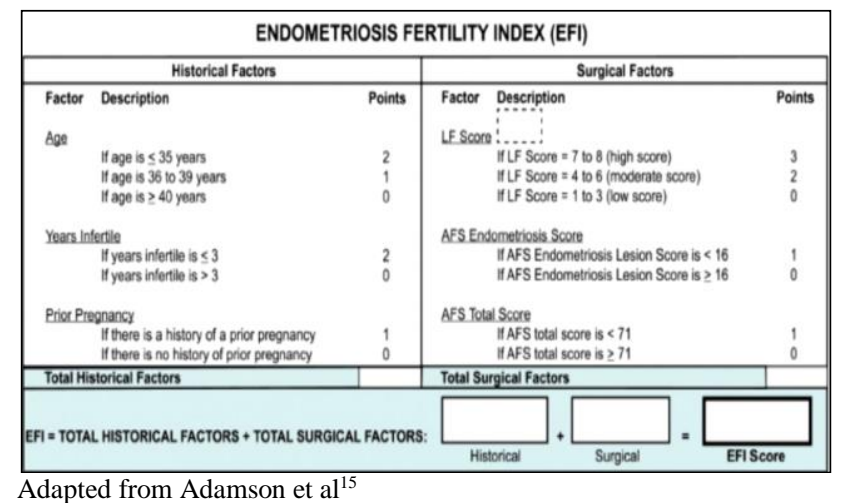

Figure 1: Calculation of EFI score. ${ }^{15}$

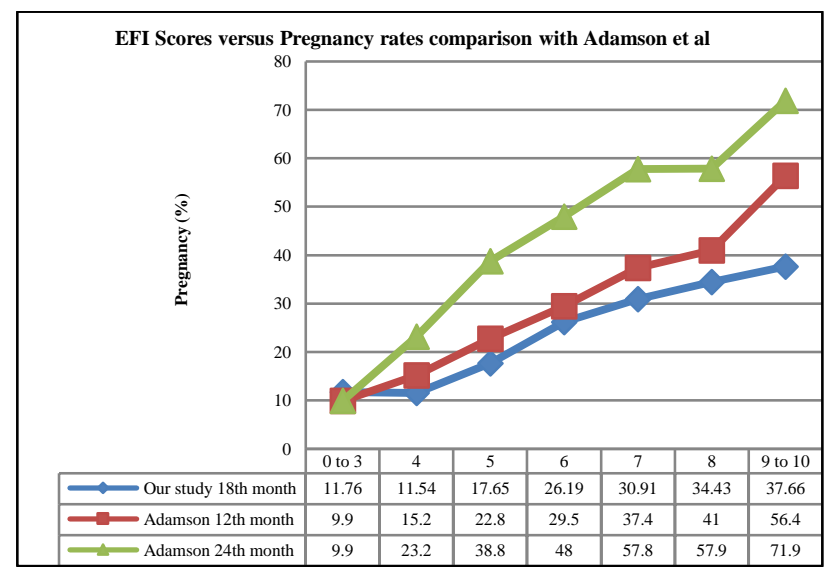

In patients with EFI score of 9 to 10 pregnancy rate observed was $37.6 \%$ at 18 months follow up. Adamson et al observed a pregnancy rate of $56.4 \%$ at 12 months and $71.9 \%$ at 24 months for score of $9-10$.

\section{Figure 2: Correlation of EFI score and pregnancy} rate.

\section{DISCUSSION}

Our study clearly demonstrated the usefulness of EFI scoring system in the prediction of pregnancy after surgery for endometriosis. We also could show significant association of pregnancy rate and LF score. The sensitivity analysis showed good correlation between LF score and EFI score as well.

The predictive power of least function score after controlling for the AFS score measures something different than AFS total score, presumably the postoperative functionality of the reproductive organs, there was high correlation between dense adhesion, especially tubal adhesions, and the least function score. The statistically significant variable used to create the EFI was the least function Score (i.e., the sum of those scores determined intra operatively after surgical intervention that describes the function of the tube, fimbria, ovary on both sides). We compared our study with David Adamson study and the results were comparable (Table 5). ${ }^{15}$ In our study, patients with EFI score of 9 to 10 had a pregnancy rate of $37.6 \%$ at 18 months follow up. In the study by Adamson et al, the pregnancy rate observed was $56.4 \%$ at a follow up of 12 months and $71.9 \%$ at 24 months follow up. The pregnancy rate was higher in the Adamson et al, study for all the subgroups of EFI scores as compared to our study. ${ }^{15}$ Hence the overall pregnancy rate as such was higher in their study and hence the absolute values may not be considered. Rather, there was an overall agreement with their study and our study. The difference, however might be due to the ethnic variations in the population and also possibly due to presence of more percentage of 
bad prognosis patients in our group. This needs to be addressed further by large multicentric and preferably prospective trials.

\section{CONCLUSION}

EFI is a simple, robust, and useful clinical tool that predicts pregnancy rate after endometriosis surgical staging. It use provides reassurance to those patients with good prognosis and avoids wasting of time and irrelevant treatment for those with poor prognosis.

Funding: No funding sources Conflict of interest: None declared

Ethical approval: The study was approved by the Institutional Ethics Committee

\section{REFERENCES}

1. American Fertility Society. Classification of endometriosis. Fertil Steril. 1979;32:633-4.

2. Guzick DS, Bross Ds, Rock JA. Assessing the efficacy of American fertility society classification of endometriosis. Fertil Steril. 1982;38:171-6.

3. Adamson GD, Frison L, Lamb EJ. Endometriosis studies of method for the design of a surgical staging system. Fertil Steril. 1982;38:659-66.

4. Buttram V. Revised Evolution of Revised American Fertility society classification of endometriosis. Fertil Steril. 1985;43:347-50.

5. American Fertility society Revised American Fertility society classification of endometriosis. Fertil Steril. 1985;43:351-2.

6. Stripling MC, Martin DC, Chatman DL, Zwaag RV, Poston WM. Subtle appearance of Pelvic endometriosis. Fertil Steril. 1988;49:427.

7. Candian GB, Vercellini P, Fedele L. Laparoscopic ovarian puncture for correct staging of endometriosis. Fertil Steril. 1990;53:994-7.
8. Vercellini P, Vendola N, Bocciolone L, Rogarinelli SG, Candiani GB. Reliability of visual diagnosis of ovarian endometriosis. Fertil Steril. 1991;56:11982000.

9. Canis M, Bouquet De, Jolinieres J, Wattiez A, Pouly JL, Mage G, et al. Classification of endometriosis. Baillieres Clin Obstet Gynaecol. 1993;7:759-74.

10. Hornstein MD, Gleason RE, ORAV J, Haas ST, Friedman AJ, Rein MS, et al. The reproducibility of the revised American Fertility society classification of endometriosis. Fertil Steril. 1993;59:1015-21.

11. Rock JA. The reproducibility of the revised American Fertility society classification of endometriosis. Fertil Steril. 1995;63:1108-10.

12. Wiegerinck MA, Van Dop PA, Brosens JA. The stage of peritoneal endometriosis by type of active lesion in addition to the revised American Fertility Society classification. Fertil Steril. 1993;60:461-4.

13. Adamson GD, Hurd SJ, Pasta DJ, Rodriguez BD. Laparoscopic endometriosis treatment. Fertil Steril. 1993;59:35-44.

14. Guzick DS, Silliman NP, Adamson GD, Buttram C, Canis M, Malinak LR, et al. Prediction of pregnancy in infertile women based on the American society for Reproductive Medicine's revised classification of endometriosis. Fertil Steril. 1997;67:822.

15. Adamson GD, Pasta DJ. Endometriosis Fertility Index: the new validated -Endometriosis staging system. Fertil Steril. 2009;94:1609-15.

Cite this article as: Louis TF, Naveen D, Ramesh P, Gopinath P, Gopinathan KK. Role of endometriosis fertility index system in predicting non-IVF conception in patients with surgically documented endometriosis. Int J Reprod Contracept Obstet Gynecol 2019;8:2363-7. 